\title{
The inverse problem concerning symmetries of ordinary differential equations
}

ARTICLE in JOURNAL OF MATHEMATICAL PHYSICS · FEBRUARY 1988

Impact Factor: $1.18 \cdot$ DOI: $10.1063 / 1.528000$

CITATIONS

2

2 AUTHORS, INCLUDING:

\section{Artemio Gonzalez-Lopez}

Complutense University of Madrid

86 PUBLICATIONS 1,723 CITATIONS

SEE PROFILE 


\title{
The inverse problem concerning symmetries of ordinary differential equations
}

\author{
F. Gonzalez-Gascon \\ Departamento de Metotos Matemáticos, Facultad de C. Fisicas, Universidad Complutense, Madrid-3, \\ Spain
}

A. González-López

Department of Physics, Princeton University, Princeton, New Jersey 08544

(Received 13 August 1987; accepted for publication 21 October 1987)

It is shown that for any local Lie group $G$ of transformations in $R \times R^{n}$ there exist differential systems of the form $\mathbf{x}^{(m}=\mathbf{f}\left(t, \mathbf{x}, \ldots, \mathbf{x}^{(m-1)}\right)$, which are symmetrical under $G$. The order $m$ of these systems is related to $\underline{r}$, the number of essential parameters of $G$.

\section{INTRODUCTION}

In a recent paper ${ }^{1}$ it was shown that for normal systems of differential equations of type

$$
\mathbf{x}^{(m}=\mathbf{f}\left(t, \mathbf{x}, \dot{\bar{x}}, \ldots, \bar{x}^{(m-1}\right) \mathbf{x} \in \mathbb{R}^{n},
$$

the maximal number of its pointlike symmetry vectors is (i) infinite, when $m=1$; (ii) not greater than $n^{2}+4 n+3$, when $m=2$; (iii) not greater than $2 n^{2}+n m+2$, when $m>2$. Since the number $2 n^{2}+n m+2$ increases without limit with $m$, the question arises of whether or not it is possible to find a system of type (1) symmetrical under a given group $G$, for a sufficiently high value of $m$. We prove that the reply to this question is affirmative. Our result is local, in the sense that the function $f$ of (1), whose existence we prove, will be, in general, only locally defined.

Note that since a first-order $(m=1)$ system always possesses an infinite number of pointlike symmetry vectors, one could naively expect to find for any $G$ a first-order system possessing $G$ among its symmetries. That this is not generally possible is seen if $G$ is, for instance, a group acting transitively on the $(t, \mathbf{x}, \dot{x})$ space. For an example see Part (1) of Sec. III.

Note also that the construction given here does not guarantee that $G$ is the maximal group of pointlike symmetries $G_{M}$ of (1), but only that $G \subset G_{M}$.

\section{MAIN RESULT}

Assume that $\mathbf{S}_{i}(t, \mathbf{x}), i=l, \ldots, r$, is a basis of generators of $G$. Calling $\mathbf{S}_{i}^{e}$ the $e$-order extension of $S_{i}$ we have ${ }^{2}$

$$
\begin{aligned}
{\left[\mathbf{S}_{i}^{(e}, \mathbf{S}_{j}^{(e}\right]=} & \sum_{k=1}^{r} c_{i j k} \mathbf{S}_{k}^{(e}, \\
& e=0,1,2, \ldots, \quad i, j=1, \ldots, r,
\end{aligned}
$$

where $c_{i j k}$ are the structure constants of $G$ associated with the basis $\left\{\mathbf{S}_{i}(t, \mathbf{x})\right\}$. On the other hand, the necessary and sufficient condition in order that $G$ be a symmetry group of equations (1) is ${ }^{2}$

$$
\left.\mathbf{S}_{i}^{(m}\left(\mathbf{x}^{(m}-\mathbf{f}\right)\right|_{\mathbf{x}^{m}=\mathbf{f}}=\mathbf{0}, \quad i=1, \ldots, r .
$$

Conditions (3) indicate that the manifold $M^{m}$ of $\left(t, \mathbf{x}, \ldots, \mathbf{x}^{(m}\right)$ space defined by Eq. (1) is invariant under the action of the vector fields $\mathbf{S}_{1}^{(m}, \ldots, \mathbf{S}_{r}^{(m)}$. We are going to prove that given $G$, one can find a sufficiently high $\underline{m}$ such that, for a certain $\mathbf{f}$, Eqs. (1) do possess $G$ as a group of symmetries.
The idea of the proof is to eliminate the possible transitivity of the action of $G^{e}$ on $D^{e}=\left\{\left(t, \mathbf{x}, \ldots, \mathbf{x}^{(e)}\right)\right\}$ for low values of $\underline{e}$ by making $e$ bigger and bigger. This is made possible, essentially, due to property (2), implying that at any point of $D^{e}$ the vector fields $\mathbf{S}_{i}^{(e}$ generate an involutive distribution $\mathscr{D}^{e}$ (Ref. 3 ) of dimension not greater than $r$. To avoid singularity points where the dimension of the distribution $\mathscr{D}^{e}$ changes value, we restrict conveniently the domain $D^{e}$ in order that in this restricted domain $\widetilde{D}^{e}, \operatorname{dim}\left(\mathscr{D}^{e}\right)$ keeps a constant and maximum value $d_{e}$. Of course $\operatorname{dim} \mathscr{D}^{e-1}=d_{e}-1$ in the projection of $\widetilde{D}^{e}$ along the $\mathbf{x}^{(e}$ axis. See Ref. 4 for details.

Therefore let $\mathbf{S}_{1}^{(e}, \ldots, \mathbf{S}_{d_{e}}^{(e}$ be a local basis of $\mathscr{D}^{e}$. Note that it might be necessary to renumber the generators of $G$ for the basis of $\mathscr{D}^{e}$ to appear in this way. Conditions (3) for the symmetry of (1) under $G$ now take the form

$$
\left.\mathbf{S}_{i}^{(m}\left(\mathbf{x}^{(m}-\mathbf{f}\right)\right|_{\mathbf{x}^{(m)}=\mathbf{f}}=\mathbf{0}, \quad i=1, \ldots, d .
$$

Writing Eq. (1) in the implicit form

$$
\mathbf{E}\left(t, \mathbf{x}, \ldots, \mathbf{x}^{(m)}\right)=0,
$$

where $\mathbf{E}$ is a vector of $m$ components, Eqs. (4) take the form

$$
\mathbf{S}_{i}^{(m}(\mathbf{E})_{\mathbf{E}=\mathbf{0}}=\mathbf{0}, \quad i=1, \ldots, d_{m} .
$$

A sufficient condition necessary for Eqs. (6) to be satisfied is that the $m$ components of the function $\mathbf{E}$ of (6) be local first integrals of $\mathbf{S}_{i}^{(m)}$, that is, if $\mathbf{E}$ satisfies

$$
\mathbf{S}_{i}^{(m}(\mathbf{E})=\mathbf{0}, \quad i=1, \ldots, d_{m} .
$$

But according to the Frobenius theorem ${ }^{3}$ the number of locally independent first integrals $I$ of an involutive distribution like $\mathscr{D}^{m}$ is $d_{I}=\operatorname{dim}\left(D^{m}\right)-d_{m}=1+n(1+m)$ $-d_{m}$. Now, since $d_{m} \leqslant r$ it follows that $d_{l} \geqslant n$ for sufficiently large $m$. Assuming $d_{1} \geqslant n$, in order to satisfy (7) it is sufficient to choose $n$ first integrals $I$ of $\mathbf{S}_{i}^{(m)}$ such that they satisfy the additional requirement

$$
\operatorname{rank}\left(\frac{\partial I_{i}}{\partial x_{k}^{(m}}\right)=n, \quad k=1, \ldots, n .
$$

Condition (8) guarantees, via the implicit function theorem, that the system of differential equations

$$
\begin{gathered}
I_{1}=C_{1}, \\
\vdots \\
I_{n}=C_{n}
\end{gathered}
$$


can be locally written in the normal form (1). The symbols $C_{1}, \ldots, C_{n}$ in Eqs. (9) are real numbers, and they appear since Eqs. (7) are clearly equivalent to

$$
\mathbf{S}_{i}^{(m}(\mathbf{E}-\mathbf{C})=\mathbf{0}
$$

for any $C \in R^{n}$. Let us see that condition (8) can be satisfied if $m$ is chosen such that

$$
\operatorname{dim}\left(\mathscr{D}^{m}\right)=\operatorname{dim}\left(\mathscr{D}^{m-1}\right) .
$$

In fact, if (8) were not satisfied by an appropriate choice of $I_{1}, \ldots, I_{n}$ between the $d_{l}$ first integrals of $\mathscr{D}^{m}$, we would have

$$
\sum_{k=1}^{n} a_{k} \frac{\partial I_{i}}{\partial x_{k}^{(m}}=0, \quad i=1,2, \ldots, d_{I},
$$

where $a_{k}$ are functions on $D^{m}$.

But (12) implies that the vector field $Z$ defined by

$$
\mathbf{Z}=\sum_{x=1}^{n} a_{k} \frac{\partial}{\partial x_{k}^{(m)}}
$$

has $I_{1}, \ldots, I_{d_{1}}$ as first integrals. Therefore $\mathbf{Z} \in \mathscr{D}^{m}$ and we can write

$$
\mathbf{Z}=\sum_{i=1}^{d_{m}} c_{i} \mathbf{S}_{i}^{(m}
$$

for certain functions $c_{i}$ defined on $D^{m}$.

Projecting (14) on the vectors

$\frac{\partial}{\partial t} ; \frac{\partial}{\partial x_{i}} ; \frac{\partial}{\partial x_{i}^{(1}}, \ldots, \frac{\partial}{\partial x_{i}^{(m-1}}, \quad \mathbf{0}=\sum_{i=1}^{d_{m}} c_{i} \mathbf{S}_{i}^{(m-1}$.

But (15) and (11) are contradictory since from the fact that $\mathbf{S}_{1}^{(m}, \ldots, \mathbf{S}_{d_{m}}^{(m)}$ are a basis of $\mathscr{D}^{m}$ it immediately follows (note that $\mathbf{S}_{i}^{m-1}$ does not depend on $\mathbf{x}^{(m)}$ that $\mathbf{S}_{i}^{(m-1}, \ldots, \mathbf{S}_{d_{m}}^{(m-1}$ generate $\mathscr{D}^{m-1}$. Hence by (16), $\operatorname{dim} \mathscr{D}^{m-1}<d_{m}$ and (12) is contradicted. Therefore (11) implies (8).

It remains only to prove that (11) can always be satisfied by choosing $m$ conveniently. But this follows from the fact ${ }^{4}$ that

$$
\operatorname{dim}\left(\mathscr{D}^{r}\right) \leqslant \operatorname{dim}\left(\mathscr{D}^{r+1}\right) .
$$

Indeed, since the dimension is a positive integer, nondecreasing by (16), and bounded by $r$ (the number of parameters of the group) it is obvious that for a certain $\underline{m}$ (12) holds. Furthermore, $m$ satisfies

$$
m \leqslant r-\operatorname{dim}\left(\mathscr{D}^{0}\right)+1=r_{1},
$$

since the worst situation that can occur concerning (11) is that

$$
\operatorname{dim}\left(\mathscr{D}^{s}\right)=\operatorname{dim}\left(\mathscr{D}^{s-1}\right)+1, \quad s<m,
$$

in which case (17) would hold with the equal sign.

Note that if we require $m$ to be greater than (or equal to) a given $k(k=1,2, \ldots)$ then the above considerations lead to the inequality

$$
m \leqslant r-\operatorname{dim}\left(\mathscr{D}^{k-1}\right)+k=r_{k}
$$

the equality sign being valid only when the sequence of dimensions $\operatorname{dim}\left(\mathscr{D}^{k-1}\right), \operatorname{dim}\left(\mathscr{D}^{k}\right), \ldots$ is strictly increasing by 1 at each step and condition (11) is fulfilled when $\operatorname{dim}\left(\mathscr{D}^{m}\right)=\operatorname{dim}\left(\mathscr{D}^{m-1}\right)=r$.
Note also that calling $r(G)$ the minimum integer such that for $k \geqslant r(G), \operatorname{dim}\left(\mathscr{D}^{k}\right)$ maintains a constant value, that is, $\operatorname{dim}\left(\mathscr{D}^{k}\right)=\operatorname{dim}\left(\mathscr{D}^{k^{\prime}}\right)$ for every $k, k^{\prime} \geqslant r(G)$, by $(11)$ we can say that for every $m>r(G)$ there are systems of differential equations of order $m$ invariant under $G$.

Note finally that [see Eq. (11)] the construction given here actually assures the existence of $n$-parameter families of systems of type (1) invariant, for any value of the parameters, under $G$. Let us now see, with two examples, that condition (11) is not necessary for the existence of individual systems of type (1) invariant under $G$.

\section{EXAMPLES SHOWING THAT CONDITION (11) IS NOT NECESSARY}

(1) Let us take as $G$ the Poincaré group in $R \times R^{2}$ (two spatial dimensions). We shall see that (11) is not satisfied either for $m=1$ or for $m=2$. Nevertheless, as has been shown elsewhere ${ }^{5} \ddot{\bar{x}}=\overline{0}$ is a second-order differential system (in fact the only one) invariant under the Poincaré group in $R \times R^{2}$. In fact the six generators of the group are

$$
\begin{aligned}
& \mathbf{S}_{1}=\frac{\partial}{\partial t} ; \quad \mathbf{S}_{1+c}=\frac{\partial}{\partial x_{i}} ; \quad \mathbf{S}_{4}=x_{1} \frac{\partial}{\partial x_{2}}-x_{2} \frac{\partial}{\partial x_{1}} \\
& \mathbf{S}_{4+i}=-\left(x_{i} \frac{\partial}{\partial t}+t \frac{\partial}{\partial x_{i}}\right) ; \quad i=1,2
\end{aligned}
$$

Since $\operatorname{dim}\left(\mathscr{D}^{\circ}\right)=3$ the group acts transitively on $D^{\circ}$ $=\left(t, x_{1}, x_{2}\right)$.

The corresponding generators of the first extension of $G$ are given by

$$
\begin{aligned}
& \mathbf{S}_{1}^{(1}=\frac{\partial}{\partial t} ; \quad \mathbf{S}_{1+i}^{1}=\frac{\partial}{\partial x_{i}} \\
& \mathbf{S}_{4}^{(1}=\mathbf{S}_{4}+\dot{x}_{1} \frac{\partial}{\partial \dot{x}_{2}}-\dot{x}_{2} \frac{\partial}{\partial \dot{x}_{1}}, \\
& \mathbf{S}_{4+i}^{(1}=\mathbf{S}_{4+i}+\left(\dot{x}_{i}^{2}-1\right) \frac{\partial}{\partial \dot{x}_{i}}+\dot{x}_{i} \dot{x}_{j} \frac{\partial}{\partial \dot{x}_{j}} .
\end{aligned}
$$

One can immediately check that $\operatorname{dim}\left(\mathscr{D}^{1}\right)=5$. Indeed, the singular points of $\mathscr{D}^{1}$ are only those satisfying $1-\dot{x}_{1}^{2}$ $-\dot{x}_{2}^{2}=0$. Therefore $\widetilde{D}_{1}=\left\{\left(t, x_{1}, x_{2}, \dot{x}_{1}, \dot{x}_{2} \mid 1-\dot{x}_{1}^{2}-\dot{x}_{2}^{2}\right.\right.$ $\neq 0\}$. Here $G^{1}$ acts transitively in each of the two unconnected components of $\widetilde{D}_{1}$ and also in the set $1-\dot{x}_{1}^{2}-\dot{x}_{2}^{2}=0$. Accordingly, it is impossible to find a single first-order system of type (1) symmetric under $G$.

The second-order extension of $G$ is defined by

$$
\begin{aligned}
& \mathbf{S}_{1}^{(2}=\frac{\partial}{\partial t} ; \quad \mathbf{S}_{1+i}^{(2}=\frac{\partial}{\partial x_{i}}, \\
& \mathbf{S}_{4}^{(2}=\mathbf{S}_{4}^{(1)}+\left(-\ddot{x}_{2} \frac{\partial}{\partial \ddot{x}_{1}}+\ddot{x}_{1} \frac{\partial}{\partial \ddot{x}_{2}}\right) \\
& \mathbf{S}_{5}^{(2}=\mathbf{S}_{5}^{(1)}+\left(3 \dot{x}_{1} \ddot{x}_{1} \frac{\partial}{\partial \ddot{x}_{1}}+\left(2 \dot{x}_{1} \ddot{x}_{2}+\dot{x}_{2} \ddot{x}_{1}\right) \frac{\partial}{\partial \ddot{x}_{2}}\right), \\
& \mathbf{S}_{6}^{(2}=\mathbf{S}_{6}^{(1}+\left(\left(2 \dot{x}_{2} \ddot{x}_{1}+\dot{x}_{1} \ddot{x}_{2}\right) \frac{\partial}{\partial \ddot{x}_{1}}+3 \dot{x}_{2} \ddot{x}_{2} \frac{\partial}{\partial \ddot{x}_{2}}\right) .
\end{aligned}
$$

We can see that $\operatorname{dim} \mathscr{D}^{2}=6$. Since $\operatorname{dim} \mathscr{D}^{2}=6$ $>\operatorname{dim}\left(\mathscr{D}^{1}\right)=5$ condition (11) is not satisfied. But from this fact one cannot conclude, in general, that there are not 
systems of type (1), for $m=2$, symmetrical under $G$. In fact the system $\ddot{\bar{x}}=0$ is a system invariant under $G$.

Note that since $\operatorname{dim} \mathscr{D}^{2}=6$ and the group has six parameters it is clear that for $k \geqslant 2$ we will have $\operatorname{dim}\left(\mathscr{D}^{k}\right)=6$. Therefore (12) is satisfied for every $m>2$ and by the results of Sec. II we can say that there are two-parameter families of $m$-order $(m>2)$ differential systems symmetrical under $G$.

(2) Let us take now as $G$ the conformal group in $R \times R$. (Note that the space is now only one dimensional.)

The six generators of this group can be taken as

$\mathbf{S}_{1}=\frac{\partial}{\partial t} ; \quad \mathbf{S}_{2}=\frac{\partial}{\partial x} ; \quad \mathbf{S}_{3}=t \frac{\partial}{\partial t}+x \frac{\partial}{\partial x}$,

$\mathbf{S}_{4}=t \frac{\partial}{\partial x}+x \frac{\partial}{\partial t} ; \quad \mathbf{S}_{5}=\left(t^{2}+x^{2}\right) \frac{\partial}{\partial t}+2 t x \frac{\partial}{\partial x}$,

$\mathbf{S}_{6}=2 t x \frac{\partial}{\partial t}+\left(t^{2}+x^{2}\right) \frac{\partial}{\partial x}$,

and therefore the third-order extension of them will be given by

$$
\begin{aligned}
\mathbf{S}_{1}^{\mathbf{u}^{3}=} & \frac{\partial}{\partial t}, \quad \mathbf{S}_{2}^{(3}=\frac{\partial}{\partial x}, \\
\mathbf{S}_{3}^{(3)}= & \mathbf{S}_{3}-\ddot{x} \frac{\partial}{\partial \ddot{x}}-2 \ddot{x} \frac{\partial}{\partial \ddot{x}}, \\
\mathbf{S}_{4}^{(3}= & \mathbf{S}_{4}+\left(1-\dot{x}^{2}\right) \frac{\partial}{\partial \dot{x}}-2 \ddot{x} \frac{\partial}{\partial \ddot{x}} \\
& +\left(-4 \dot{x} \ddot{x}-3 \ddot{x}^{2}\right) \frac{\partial}{\partial \ddot{x}}, \\
\mathbf{S}_{5}^{(3}= & \mathbf{S}_{5}+\left(2 x-2 x \dot{x}^{2}\right) \frac{\partial}{\partial \dot{x}} \\
& +\left(2 \dot{x}-2 \dot{x}^{3}-2 \ddot{x} t-6 x \dot{x} \ddot{x}\right) \frac{\partial}{\partial \ddot{x}} \\
& +\left\{-6 \ddot{x}\left(2 \dot{x}^{2}+x \ddot{x}\right)-4 \dddot{x}(t+2 x \dot{x})\right\} \frac{\partial}{\partial \ddot{x}}, \\
\mathbf{S}_{6}^{(3)=} & \mathbf{S}_{6}+2 t\left(1-\dot{x}^{2}\right) \frac{\partial}{\partial \dot{x}} \\
& +\left\{2\left(1-\dot{x}^{2}\right)-2 \ddot{x}(x+3 t \dot{x})\right\} \frac{\partial}{\partial \ddot{x}} \\
& +\{-6 \ddot{x}(2 \dot{x}+t \ddot{x})-4 \dddot{x}(x+2 t \dot{x})\} \frac{\partial}{\partial \ddot{x}} .
\end{aligned}
$$

From (24) it follows immediately that

$\operatorname{dim} \mathscr{D}^{0}=2 ; \quad \operatorname{dim} \mathscr{D}^{1}=3 ; \quad \operatorname{dim} \mathscr{D}^{2}=4 ; \quad \operatorname{dim} \mathscr{D}^{3}=5$.

We see in (25) that condition (11) is not fulfilled for $m=1$, $m=2$, and $m=3$. This implies, as we shall prove in Sec. IV, that there are no one-parameter families of differential equations of first-, second-, or third-order invariant under this group. Nevertheless, as we show now, there is one (and only one) third-order differential equation invariant under $G$.

Indeed, invariance under $S_{1}$ and $S_{2}$ implies that the third-order equation will have the form

$$
\dddot{x}=f(\dot{x}, \ddot{x}) \text {. }
$$

Invariance under $\mathbf{S}_{3}$ implies

$$
\ddot{x} \frac{\partial f}{\partial \ddot{x}}=2 f,
$$

that is,

$$
f=a(\dot{x}) \ddot{x}^{2},
$$

where $a(\dot{x})$ is an arbitrary function.

Invariance under $S_{4}$ implies

$$
\left(1-\dot{x}^{2}\right) \frac{d a}{d \dot{x}}-6 \dot{x} a=-4 \dot{x} a-3,
$$

and therefore,

$$
a(\dot{x})=(3 \dot{x}-b) /\left(\dot{x}^{2}-1\right) .
$$

Finally invariance under $S_{5}$ implies $b=0$. The resulting third-order differential equation is automatically invariant under $\mathbf{S}_{6}$.

Therefore we have obtained the differential equation

$$
\dddot{x}=\frac{3 \dot{x} \ddot{x}^{2}}{\dot{x}^{2}-1},
$$

which is the only one invariant under the conformal group in $R \times R$.

Note that since $\mathscr{D}^{1}$ does not act transitively on the whole $(t, x, \dot{x})$ space it is possible to have also first-order differential equations invariant under $G$. This is precisely what happens with the two differential equations

$$
\dot{x}=1, \dot{x}=-1,
$$

which are the only ones (of first order) invariant under $G$.

Observe that the set $\left\{(t, x, \dot{x}) \mid 1-\dot{x}^{2}=0\right\}$ defines the singular points of $\mathscr{D}^{1}$, that is, the points where $\mathscr{D}^{1}$ has (in this case) dimension 2.

Although $\mathscr{D}^{2}$ also has singular points, where its dimension does not attain the maximum value, it is easy to check by direct computation that there do not exist second-order equations invariant under the conformal group in $R \times R$. (The generators $S_{1}^{2}, S_{2}^{2}, S_{3}^{2}, S_{4}^{(2}$ imply $\ddot{x}=0$, but this equation is incompatible with the two generators $\mathbf{S}_{5}^{(2}$ and $\mathbf{S}_{6}^{(2)}$.)

\section{IV. n-PARAMETER FAMILIES OF EQUATIONS INVARIANT UNDER $G$}

The above examples show that condition (11) is, in general, not necessary for the existence of isolated systems of order $m$ invariant under $G$. We prove here that $(11)$ is necessary and sufficient for the existence of $n$-parameter families of equations, each of them being invariant under $\boldsymbol{G}$.

In fact, the necessary and sufficient conditions in order that the $n$-parameter family defined by

$$
\mathbf{E}\left(t, \bar{x}, \dot{\bar{x}}, \ldots, \bar{x}^{(m)}\right)=\bar{c}, \quad \operatorname{det}\left(\frac{\partial \bar{E}}{\partial \bar{x}^{(m}}\right) \neq 0,
$$

be invariant under $G$ are

$$
\mathbf{S}_{i}^{(m}(\mathbf{E})_{\mid \mathbf{E}=\mathbf{C}}=\mathbf{0}, \quad i=1, \ldots, d_{m} .
$$

Since Eqs. (34) hold as identities in $C \in R^{n}$ we must have

$$
\mathbf{S}_{i}^{(m}(\mathbf{E})=\mathbf{0} \text {. }
$$

Let us see that (35) and

$$
\operatorname{dim}\left(\mathscr{D}^{m-1}\right)<\operatorname{dim}\left(\mathscr{D}^{m}\right)
$$

are contradictory. 
In fact, as explained in Sec. II, if $\mathbf{S}_{1}^{(m}, \ldots, \mathbf{S}_{d_{m}}^{(m)}$ is a basis of $\mathscr{D}^{m}$ then $\mathbf{S}_{1}^{(m-1}, \ldots, \mathbf{S}_{d_{m}-1}^{(m-1}$ is a basis of $\mathscr{D}^{m-1}$. Therefore if (36) holds we must have

$$
\sum_{i=1}^{d_{m}} c_{i}\left(t, \mathbf{a}, \ldots, \mathbf{a}^{(m-1)}\right) \mathbf{S}_{i}^{(m-1}=\mathbf{0},
$$

where not all of the $c_{i}$ are equal to zero.

Therefore

$$
\sum_{i=1}^{d_{m}} c_{i} \mathbf{S}_{i}^{(m)}(\mathbf{E}) \stackrel{(37)}{=} \sum_{i=1}^{d_{m}} c_{i} \sum_{j=1}^{n} \psi_{i j}^{(m} \frac{\partial(\mathbf{E})}{\partial x_{j}^{(m}} \stackrel{(35)}{=} \mathbf{0}
$$

contradicting the hypothesis of $\operatorname{det}\left(\partial \mathrm{E} / \partial \mathbf{x}^{(m)}\right) \neq 0$ imposed in (33). Note that this hypothesis concerning the determinant is essential in order to be able to apply the implicit function theorem to the variables $x_{1}^{(m}, \ldots, x_{n}^{(m}$ and put Eqs. (33) in the normal form (1). To conclude, we give an example of a one-parameter family of second-order differential equations invariant under the Poincaré group in $R \times R$.

In this case, the generators of $G$ can be taken as

$$
\mathbf{S}_{1}=\frac{\partial}{\partial t}, \quad \mathbf{S}_{2}=\frac{\partial}{\partial x}, \quad \mathbf{S}_{3}=x \frac{\partial}{\partial t}+t \frac{\partial}{\partial x} .
$$

The first and second extensions are given by

$$
\mathbf{S}_{1}^{(1)}=\frac{\partial}{\partial t} ; \quad \mathbf{S}_{2}^{(1)}=\frac{\partial}{\partial x} ; \quad \mathbf{S}_{3}^{(1}=\mathbf{S}_{3}+\left(1-\dot{x}^{2}\right) \frac{\partial}{\partial \dot{x}},
$$

and

$$
\mathbf{S}_{1}^{(2)}=\frac{\partial}{\partial t} ; \quad \mathbf{S}_{2}^{(2)}=\frac{\partial}{\partial x} ; \quad \mathbf{S}_{3}^{(2}=\mathbf{S}_{3}^{(1}+(-3 \dot{x} \ddot{x}) \frac{\partial}{\partial \ddot{x}} .
$$

One can immediately see that

$$
\operatorname{dim} \mathscr{D}^{0}=2, \quad \operatorname{dim} \mathscr{D}^{1}=3 .
$$

Since the group has three parameters it is clear that $\operatorname{dim} \mathscr{D}^{k}=3$ for any $k \geq 1$. Therefore there are one-parameter families of differential equations of order $m$ (for any $m \geqslant 2$ ) invariant under this group. Taking for simplicity $m=2$, the symmetry of the equation

$$
\ddot{x}=f(t, x, x)
$$

under $\mathbf{S}_{1}$ and $\mathbf{S}_{2}$ implies

$$
\frac{\partial f}{\partial t}=0 ; \quad \frac{\partial f}{\partial x}=0
$$

that is, $f(t, x, x)=g(\dot{x})$. The symmetry under the boosts $S_{3}$ implies

$$
-3 \dot{x} g=\frac{d g}{d \dot{x}}\left(1-\dot{x}^{2}\right)
$$

which leads, after integration, to the one-parameter family

$$
\ddot{x}=c\left(1-\dot{x}^{2}\right)^{3 / 2} \text {. }
$$

'F. González-Gascón and A. González-López, J. Math. Phys. 24, 2006 (1983).

${ }^{2} \mathbf{P}$. Olver, Applications of Lie Groups to Differential Equations (Springer, New York, 1986).

${ }^{3} \mathrm{~F}$. Warner, Foundations of Differential Geometry and Lie Groups (Freeman, San Francisco, 1971).

${ }^{4} A$. González-López, Ph.D. thesis, Universidad Complutense, Madrid, 1984 (unpublished).

${ }^{5}$ F. González-Gascón and A. González-López, Hadronic J. 6, 841 (1983). 